\title{
Effect of nitazoxanide on cryptosporidiosis in experimentally infected neonatal dairy calves
}

\author{
T. L. Ollivett, ${ }^{*}$ D. V. Nydam, $\dagger^{1}$ D. D. Bowman,ł J. A. Zambriski, $\dagger$ M. L. Bellosa, $†$ T. C. Linden, $\dagger$ and T. J. Divers* \\ *Department of Clinical Sciences, Box 20, College of Veterinary Medicine, Cornell University, Ithaca, NY 14853 \\ †Department of Population Medicine and Diagnostic Sciences, Box 29, College of Veterinary Medicine, Cornell University, Ithaca, NY 14853 \\ ‡Department of Microbiology and Immunology, C5-181 VMC, College of Veterinary Medicine, Cornell University, Ithaca, NY 14853
}

\section{ABSTRACT}

Cryptosporidium is a zoonotic protozoan that is most often diagnosed in association with diarrhea in 1- to 3 -wk-old dairy calves. There are neither consistently effective nor approved antimicrobial drugs for treatment in animals. The objective of this study was to test nitazoxanide (NTZ) as a treatment for cryptosporidiosis in experimentally infected dairy calves. A randomized, controlled, and blinded trial was performed using Holstein bull calves obtained from a large commercial dairy. All births were attended by study personnel and calves were fed $4 \mathrm{~L}$ of heat-treated colostrum within 1 $\mathrm{h}$ of birth. Calves were randomly assigned to treatment or placebo group and maintained for a 32-feeding (16 d) study period. Twenty-three calves were enrolled with 3 lost to follow up. Thirteen calves were assigned to the treatment group and 7 calves to the placebo group. All calves were inoculated with $1 \times 10^{6}$ viable Cryptosporidium parvum oocysts at feeding 3 . Treatment was a commercially available NTZ product and the placebo was the carrier of the same product. Nitazoxanide was administered at $1.5 \mathrm{~g}$ twice per day for $5 \mathrm{~d}$. Nitazoxanide or placebo treatment began after feeding 10 and when the fecal score was greater than 1 out of 3 . Outcome measurements included twice-daily fecal and health scores and a once-daily oocyst count by an immunofluorescent antibody assay. Data were analyzed by nonparametric and time-to-event methods. Measures of passive transfer of antibodies, initial body weight, and onset of oocyst shedding were not different between treatment and control calves. Eighty-five percent of the NTZ-treated calves stopped shedding oocysts by the end of the observation period whereas only $15 \%$ of the placebo group stopped shedding. The median number of feedings with a fecal score equal to 3 was 2 in the NTZ group while it was 6 in the placebo group. Calves receiving NTZ were 0.13 times as likely to have severe and sustained diarrhea than control calves (95\% con-

Received June 20, 2008.

Accepted November 20, 2008.

${ }^{1}$ Corresponding author: dvn2@cornell.edu fidence interval, 0.02-0.98). Treating calves with NTZ reduced the duration of oocyst shedding and improved fecal consistency.

Key words: nitazoxanide, cryptosporidiosis, diarrhea, dairy calf

\section{INTRODUCTION}

Cryptosporidium parvum is a zoonotic organism that is the pathogen most often diagnosed in association with diarrhea, dehydration, and death in young, 1- to 3-wkold dairy calves (Moore et al., 2003; Trotz-Williams et al., 2007). It has been estimated that approximately 60 to $90 \%$ of dairy herds in North America have at least one calf infected with this organism (Garber et al., 1994; Trotz-Williams et al., 2008). Health of infected calves ranges from being clinically normal with fecal shedding, to having severe diarrhea, anorexia, and secondary dehydration (Moore and Zeman, 1991; Jarvie et al., 2005).

Infection with $C$. parvum usually occurs via fecal-oral transmission, either directly or through contaminated water. Oocysts can survive for several weeks to months in the environment and likely cause infection when calves are born into contaminated maternity pens or housed within contaminated calf hutches or pens (Anderson, 1998). This organism is a protozoal parasite that undergoes sexual and asexual reproduction and may complete its life cycle within the host animal via thin-walled oocysts (Anderson, 1998). Readily infective thick-walled oocysts, containing 4 sporozoites each, are then shed from infected calves. Once ingested, sporozoites invade the microvillus brush border of the small intestinal (and occasionally, colonic) enterocytes, which causes destruction of the adjacent enterocyte and villus atrophy, villus fusion, and intestinal crypt inflammation. This intestinal pathology creates a malabsorptive, maldigestive, and osmotic diarrhea that may or may not be secretory in nature (Saini et al., 2000; Elitok et al., 2005). The severity of this diarrhea and its subsequent dehydration influences the morbidity and mortality of the calf (O'Handley et al., 1999). 
On-farm management goals for the control of cryptosporidiosis primarily focus on cleanliness of maternity pens, calf housing and feeding equipment, separation of dam and calf at birth, as well as early detection of anorexia, diarrhea, and dehydration in neonatal calves (Harp and Goff, 1998; Nydam and Mohammed, 2005). Treatment is supportive, focusing on prevention and correction of fluid and energy deficits and electrolyte disturbances associated with the diarrhea. There is neither an approved product nor an extra-label product available in the United States that has shown consistent efficacy toward reducing $C$. parvum-associated diarrhea or fecal shedding of oocyst in dairy calves (Harp and Goff, 1995; Nydam and Peregrine, 2005).

Nitazoxanide (NTZ), a member of the thiazolide drug class, has been shown to reduce the duration of diarrhea and oocyst shedding of $C$. parvum in human adults (500 $\mathrm{mg}$ oral, twice daily for $3 \mathrm{~d}$ ) and children (8 $\mathrm{mg} / \mathrm{kg}$ oral, twice daily for $3 \mathrm{~d}$ ) without immunodeficiencies (Rossignol, 2006). Higher doses (1,000 mg oral, twice daily for $14 \mathrm{~d}$ ) have been shown to be effective in resolving diarrhea and eliminating oocysts from feces in patients with acquired immune deficiency syndrome with CD4 counts $>50$ cells $/ \mathrm{mm}^{3}$ (Rossignol, 2006; Abubakar et al., 2007). Nitazoxanide has also shown efficacy against C. parvum in goats (Viel et al., 2007). Nitazoxanide is also currently available in the United States as a treatment for equine protozoal myelitis (Navigator paste, Idexx Pharmaceuticals Inc., Greensboro, NC). The mechanism of its anticryptosporidial action is unknown at this point. The objective of this study was to determine the effect of NTZ on cryptosporidiosis in experimentally challenged dairy calves.

\section{MATERIALS AND METHODS}

\section{Challenge Model/Treatment Solutions and Administration}

Calves used in this study were cared for in compliance with the Institutional Animal Care and Use Committee (IACUC) of Cornell University. This randomized, placebo-controlled, double-blinded study was performed at the College of Veterinary Medicine, Cornell University (Ithaca, NY). From June 2007 through August 2007, 23 bull calves were purchased at birth from a local dairy farm and enrolled in the study as they were born. At least one study author attended all calvings. The perineum of the dam was thoroughly cleaned with povidone-iodine scrub and calves were caught on single-use plastic to prevent on-farm manure contamination. The calves were then transported to a designated vehicle designed to hold 3 calves in individual pens, be thoroughly washable, and safe for both the animals and the operator of the vehicle. Immediately after birth, a physical examination was performed and an identification tag was placed in the right ear. Within $1 \mathrm{~h}$ of birth, $4 \mathrm{~L}$ of warm, heat-treated (Dairy Tech Inc., Windsor, CO) colostrum from a single dam was fed via an esophageal tube feeder, as has been previously validated (Johnson et al., 2007). Upon arrival from the source farm, calves were individually housed in permanent $0.91 \times 1.83 \mathrm{~m}$ stalls with high tile walls and concrete flooring within a closed barn with an active ventilation system. This prevented contact between calves. Calves were fed commercial $22 \%$ protein $/ 20 \%$ fat nonmedicated milk replacer (Nursing Formula NT Calf Milk Replacer, Land O'Lakes Inc.) at $0.68 \mathrm{~kg}$ of DM per day, split into 2 feedings, for the duration of the study. Any milk replacer not consumed within 15 min was fed via an esophageal tube feeder. Free-choice water was available at all times. Stalls were cleaned and bedded daily with pine shavings and steam cleaned with $93^{\circ} \mathrm{C}\left(200^{\circ} \mathrm{F}\right)$ pressurized water between calves. Gross manure contamination on the stall walls was also removed daily. Pull-over boots, water buckets, milk buckets, milk bottles, and thermometers were labeled for each calf and not shared between calves.

Calves were enrolled in the study for 32 feedings. They were randomized by a number generator to either the NTZ treatment group or the placebo group from birth to achieve an approximately 2 to 1 ratio of treated to control calves. Each calf was inoculated with a $>90 \%$ viable, as determined using a dye permeability assay (Jenkins et al., 1997), field strain of C. parvum at a dose of $1 \times 10^{6}$ oocysts (Peeters et al., 1993). Inoculation occurred $1 \mathrm{~h}$ after feeding 3 as long as serum total protein (TP) measured by refractometer was $5.0 \mathrm{mg} /$ dL or greater. Five milliliters of oocyst suspension was delivered orally via the rigid oral portion of an esophageal feeder. Water $(120 \mathrm{~mL})$ was then flushed through the feeder to ensure all of the oocyst suspension was delivered to the calf.

All study personnel making calf-level observations were blinded to treatment group. At each feeding, the following were recorded: health score, fecal score (FS), and temperature. Health scores were based on a 4-point scale; $1=$ normal, $2=$ mildly depressed, $3=$ severely depressed, $4=$ moribund or dead. Fecal scores were based on a 3 -point scale; 1 = sample is in "patty" form; minimal water content, does not flow across or down a surface; $2=$ sample is more of a puddle, some water content, flows slowly across or down a surface; $3=$ sample is watery, flows across or down a surface while leaving some to no adherent material (Moore et al., 2003). Severe, sustained diarrhea was defined as more than one quarter of fecal scores equal to $\mathrm{FS}=3$. Fecal samples were obtained at every even-numbered feeding 
after inoculation with $C$. parvum for oocyst quantification and dry weight measurements of positive samples. Serum TP was measured by refractometer at feeding 3 (Kernco Instruments Co., El Paso, TX). Body weight was measured with a digital scale at feedings 3,16 , and 32 .

Both NTZ and placebo treatments were initiated at feeding number greater than 10 and fecal score greater than 1 out of 3 . These criteria were chosen because the prepatent period is 5 to $7 \mathrm{~d}(\sim 10-14$ feedings) and we wanted to treat clinical cases. Treatments were prepackaged into ten $60-\mathrm{mL}$ oral dosing syringes per calf. Each syringe was labeled with calf ID and dose number. A 4.7-g oral twice-daily dose of commercial NTZ vehicle or the commercial NTZ product itself (containing $320 \mathrm{mg}$ of NTZ per g of product; equivalent to a 1.5 $\mathrm{g}$ of oral, twice-daily dose per calf) were weighed out on a digital scale and placed into dosing syringe. Just before feeding, $30 \mathrm{~mL}$ of warm water was added to the oral dosing syringe and the mixture was thoroughly shaken until a complete suspension was formed. There was no visual difference between the NTZ suspension and the placebo control suspension. This suspension was then added to $1 \mathrm{~L}$ of milk replacer at $43.3^{\circ} \mathrm{C}$ in a nipple bottle, thoroughly shaken, and fed to the calf. The remaining $1 \mathrm{~L}$ of that feeding's milk replacer, not containing NTZ or placebo treatments, was fed via a bucket. Control calves were sold and treatment calves killed via penetrating captive bolt and exsanguination at the termination of the study.

\section{Fecal Sample Analysis}

Quantitative analysis of C. parvum oocysts in the fecal samples collected was performed using Merifluor Crypto/Giardia immunofluorescence antibody detection reagent from Meridian Diagnostics (Cincinnati, $\mathrm{OH}$ ). The procedure used for immunofluorescence was a modification from the kit instructions. A 0.10 -g portion of feces was mixed into $10 \mathrm{~mL}$ of PBS $(\mathrm{pH}=7.4)$ in a 15 $\mathrm{mL}$ conical centrifuge tube. Then, $100 \mu \mathrm{L}$ of the mixture was removed and $5 \mu \mathrm{L}$ of Merifluor immunofluorescence antibody reagent was added. This solution was vortexed then incubated in the dark at room temperature for at least $30 \mathrm{~min}$ and stored at $4^{\circ} \mathrm{C}$ until examination. Once incubated, a $10.5-\mu \mathrm{L}$ sample was placed on a slide and covered with a coverslip. The $10 \times$ objective on a fluorescent compound binocular microscope (460-490 wavelength fluorescent compound binocular microscope Olympus BX41, Olympus America Inc., Center Valley, PA) was used to read the slide and count the number of oocysts observed. The number of oocysts observed in $10.5 \mu \mathrm{L}$ is then multiplied by 10,000 to give the number of oocysts per gram of feces. This was then standard- ized by the dry weight percentage. Dry weight analysis of fecal samples was obtained by taking a 10- to $20-\mathrm{g}$ portion of each original fecal sample, drying it at $108^{\circ} \mathrm{C}$ for a minimum of $24 \mathrm{~h}$ (Thermolyne Mechanical Oven, Barnstead International, Dubuque, IA), then weighing it directly (Precision Standard Scale, Ohaus Corporation, Pine Brook, NJ).

\section{Analysis}

Data were analyzed using descriptive and inferential methods. Continuous data were described by medians and interquartile ranges and categorical data were summarized by contingency tables. Comparisons of continuous data between calves treated with NTZ and placebo-treated calves were analyzed using Wilcoxon rank sum tests because these data often had nonnormal distributions (Rosner, 1986). Categorical data were analyzed using and chi-squared or Fisher's exact tests with calculation of relative risk and confidence intervals (Dean et al., 1994). Time-to-event data (i.e., days to cessation of oocyst shedding after treatment commenced) was estimated using the Kaplan-Meier product limit method (Kaplan and Meier, 1958) and ADG of weight was estimated for each calf using simple linear regression. Data were analyzed using commercially available software (SAS Institute Inc., Cary, NC).

\section{RESULTS}

Three calves were replaced in the study: 1 was killed at $2 \mathrm{~d}$ of age due to cerebellar dysfunction, 1 Jersey cross-breed was considerably smaller than the other calves, and 1 calf missed its second treatment dose. Of the 20 Holstein bull calves that completed the trial, 13 were in the NTZ treatment group and 7 were in the placebo control group after randomization. All calves willingly drank their treatments from a bottle, shed oocysts, and had clinical diarrhea at some point throughout the study.

Passive transfer of maternal antibodies ( $\mathrm{TP}=5.6$ vs. $5.65 \mathrm{~g} / \mathrm{dL} ; P=0.93$ ), BW at feeding 3 (48.6 vs. 46.8 kg; $P=0.8)$, and onset of oocyst shedding ( 7 vs. $7 \mathrm{~d} ; P$ $=0.68$ ), between treatment and control calves, respectively, were not different, indicating that these potential confounders did not affect our outcomes. No differences existed in peak number of oocysts shed $\left(1.74 \times 10^{7} \mathrm{vs}\right.$. $\left.2.02 \times 10^{7} ; P=0.63\right)$, total oocysts counted during the posttreatment observation period $\left(3.51 \times 10^{7}\right.$ vs. 3.87 $\times 10^{7} ; P=0.88$ ), health scores ( 1 vs. $1 ; P=0.8$ ), nor time to first treatment ( 8.5 vs. $9 \mathrm{~d} ; P=0.60$ ) between treatment and control calves, respectively (Table 1 ).

Differences that did occur between treatment and control calves included duration of oocyst shedding, 


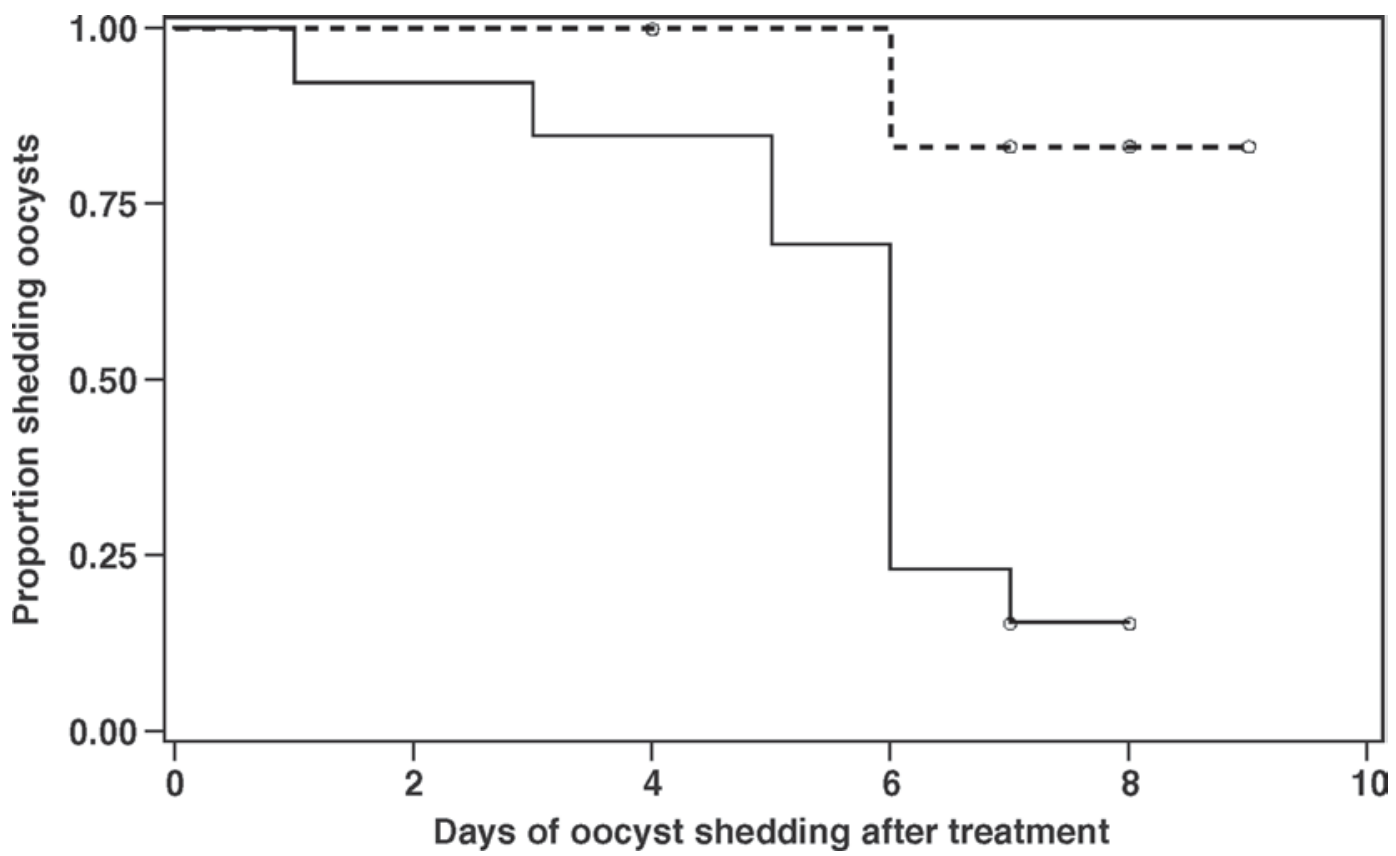

Figure 1. Kaplan-Meier survival curves for time (d) to cessation of oocyst shedding from initiation of treatment in placebo-treated calves (dashed line) and calves treated with nitazoxanide (NTZ; solid line). Fifteen percent of the NTZ-treated calves failed to stop shedding, whereas $85 \%$ of the placebo-treated calves were shedding oocysts by the end of the observation period $(P=0.01)$.

cessation of oocyst shedding, and severity of diarrhea. Eighty-five percent of the NTZ-treated calves finished shedding by the end of the observation period, whereas $15 \%$ of the placebo-treated calves finished shedding $(P$ $=0.01$; Figure 1 ). Median days of oocyst shedding was $6 \mathrm{~d}$ for NTZ-treated calves whereas control calves shed for $7 \mathrm{~d}(P=0.07)$. After initiation of treatment, the median number of feedings with fecal score $=3$ was 2 and 6 for the NTZ-treated and placebo-treated calves, respectively $(P=0.06$; Figure 2$)$. Calves receiving NTZ were 0.13 times as likely to have severe, sustained diarrhea (95\% CI: $0.02-0.98 ; P=0.01)$.

\section{DISCUSSION}

In this study, our challenge model successfully created clinical cryptosporidiosis. Commercially available NTZ was highly palatable with no clinical signs of toxicity at the oral dose of $1.5 \mathrm{~g}$ twice daily per calf, and NTZ effectively reduced the duration of oocyst shedding and severity of calf diarrhea in neonatal dairy calves experimentally infected with $C$. parvum. Among the NTZ-treated calves, $85 \%$ stopped shedding oocysts by the end of the observation period, whereas only $15 \%$ of the placebo-treated calves had stopped. This may mean the true median days of shedding in the placebo-treated calves was greater than the 7 observed days, whereas the true median days of shedding in the NTZ-treated calves was likely similar to the observed days. Of note, a few calves treated with NTZ had mild yellow staining of their milk bottles, hair coat, and urine of no apparent consequence.

Previous studies evaluating chemotherapeutics such as halofuginone, azithromycin, decoquinate, paromo-

Table 1. Results between nitazoxanide-treated and placebo-treated control calves

\begin{tabular}{lccccc}
\hline Item & $\begin{array}{c}\text { Treatment } \\
(\text { median })\end{array}$ & $\begin{array}{c}\text { Interquartile } \\
\text { range }\end{array}$ & $\begin{array}{c}\text { Control } \\
\text { (median) }\end{array}$ & $\begin{array}{c}\text { Interquartile } \\
\text { range }\end{array}$ & $P$-value \\
\hline Total protein at & & & & & \\
feeding 3, g/dL & 5.6 & 0.3 & 5.65 & 0.6 & 0.93 \\
BW at feeding 3, kg & 48.6 & 8 & 46.8 & 14 & 0.8 \\
Onset of oocyst shedding, d & 7 & 2 & 7 & 0 & 0.68 \\
Days until first treatment & 8.5 & 1.5 & 9 & 1.5 & 0.60 \\
Peak oocysts & $1.74 \times 10^{7}$ & $2.5 \times 10^{7}$ & $2.02 \times 10^{7}$ & $3.3 \times 10^{7}$ & 0.63 \\
Total oocysts & $3.51 \times 10^{7}$ & $6.6 \times 10^{7}$ & $3.87 \times 10^{7}$ & $7.6 \times 10^{7}$ & 0.88 \\
ADG, kg & $0.68^{1}$ & $0.5^{2}$ & 0.72 & $0.4^{2}$ & 0.86 \\
\hline
\end{tabular}

${ }^{1}$ Average, not median.

${ }^{2} \mathrm{SD}$, not interquartile range. 


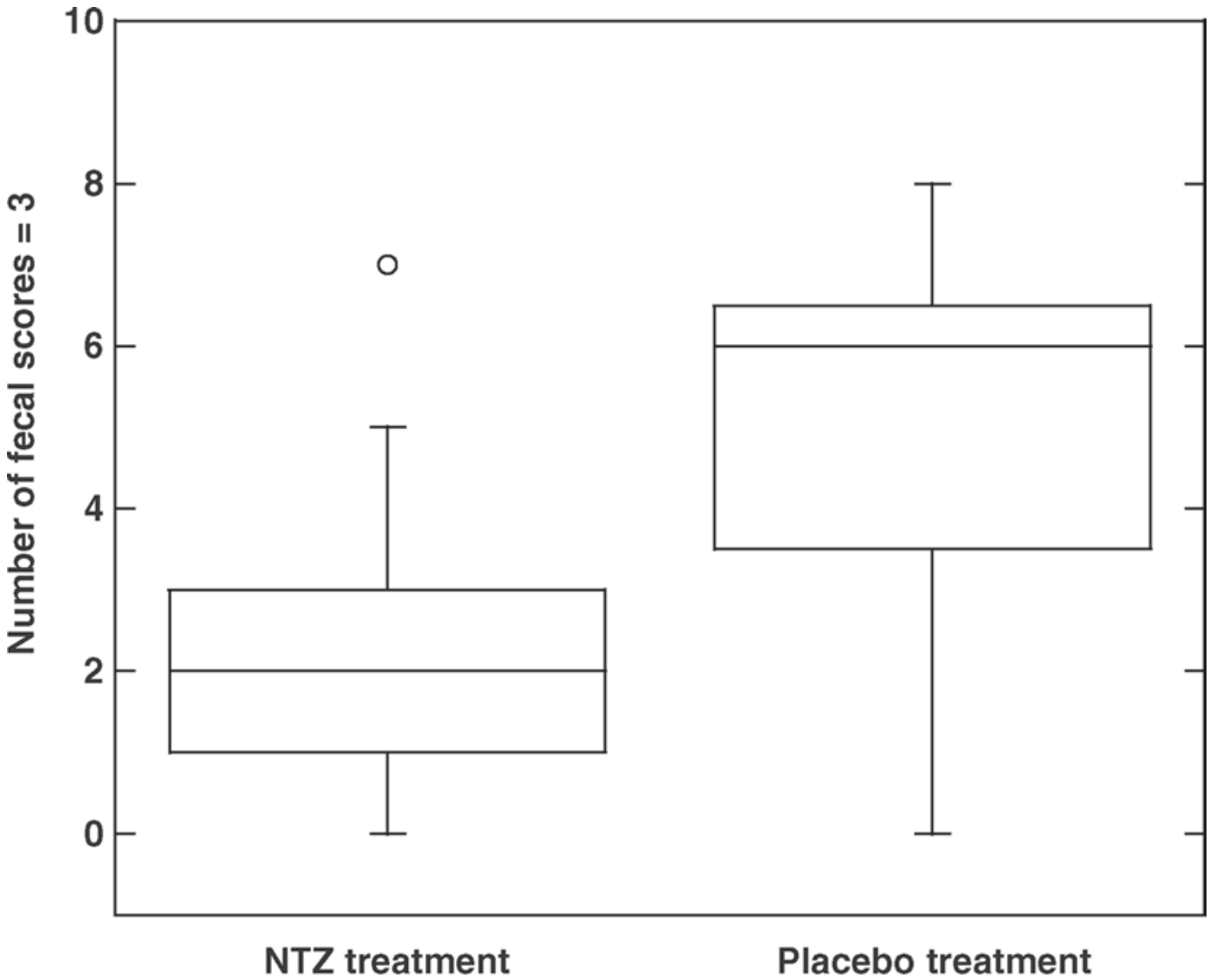

Figure 2. Box and whisker plot of number of feedings with fecal score (FS) equal to 3 (i.e., watery diarrhea). After initiation of treatment with nitazoxanide (NTZ), the median number of feedings with FS $=3$ was 2 for the NTZ-treated calves and 6 for placebo-treated calves $(P=$ $0.06)$.

mycin, and even NTZ have shown various positive and negative effects on cryptosporidiosis in neonatal calves, lambs, goat kids, and human patients. Similar to our results in calves experimentally inoculated with NTZ (Figures 1 and 2), oral treatment with halofuginone, a synthetic quinazolinone, reduced the incidence of severe diarrhea during natural infection in neonatal dairy calves (Lefay et al., 2001; Jarvie et al., 2005). However, halofuginone is not available in the United States, and is only available in Canada as a limited, emergency release product. Azithromycin, a macrolide antibiotic, was also shown to decrease oocyst shedding and reduce the severity of clinical signs associated with cryptosporidiosis in naturally infected dairy calves in Turkey; however, it is expensive to use on commercial dairy farms (Elitok et al., 2005; Nydam and Peregrine, 2005). Treatment with decoquinate, an oral coccidiostat, was shown to have no effect on clinical or laboratory findings when used on experimentally challenged calves, despite anecdotal efficacy (Moore et al., 2003). Paromomycin, an aminoglycoside antibiotic, did reduce oocyst output and clinical signs in neonatal lambs; however, detrimental effects on growth at certain dosages were noted, unlike our average daily gain findings with NTZ (Table 1; Viu et al., 2000). A study by Viel et al. (2007) showed that NTZ reduced oocyst shedding and reduced severity in neonatal experimentally challenged goat kids; however, severe toxicity resulting in death was noted in some of the study subjects. The use of NTZ in both immunocompromised and immunocompetent human patients reduced oocyst shedding and severity of diarrhea (Rossignol, 2006) consistent with our findings. It should be noted that the commercially available NTZ product is not specifically labeled for use in cattle. However, veterinarians are allowed to prescribe certain approved animal and human drugs such as NTZ in an extra-label manner under the Animal Medicinal Drug Use and Clarification Act of 1994, provided violative residues are avoided and the commercial paste is mixed only in water or oral electrolyte solutions or administered directly from the dosing syringe, as extra-label use does not apply to feed additives.

Additional studies evaluating NTZ may be useful. Such studies might include an assessment of the effect of NTZ on the occurrence of $C$. parvum infection when concurrent infection with another organism such as 
Salmonella, Escherichia coli, or rotavirus is present, as might occur in field situations, as well as the prophylactic/metaphylactic effects on C. parvum infection.

\section{CONCLUSIONS}

Nitazoxanide was shown to reduce the duration of shedding C. parvum oocysts and the severity of diarrhea in calves experimentally infected with $C$. parvum. Calf caregivers could be responsible for fewer doses of oral, intravenous, or subcutaneous electrolyte solutions, which will save time and money for the farm. Shedding of immediately infective oocysts may cause reinfection of the sick calf or infection of neighboring calves, and also poses ground water issues and health risks for the calf caregivers and visitors to the calf facility (Saini et al., 2000; Nydam et al., 2005; Collick et al., 2006). Reduction in duration of oocyst shedding will reduce the environmental pathogen load and reduce exposure of other animals and people.

\section{ACKNOWLEDGMENTS}

The authors thank the technical support of the following Cornell students participating in the Food Animal Medicine Experience program and staff for their calf-care and data-recording skills: Sarah Guest, Andrew Chambers, Amy Rauf, John Cooley, Matt Curler, Loralee Howell, and Glenn Palmer. Fred Hess, the W. C. Rebhun Memorial Fund, and Dorsey Kordick (Idexx Pharmaceuticals, Greensboro, NC) also assisted in supporting this study.

\section{REFERENCES}

Abubakar, I., S. H. Aliyu, C. Arumugam, N. K. Usman, and P. R. Hunter. 2007. Treatment of cryptosporidiosis in immunocompromised individuals: Systematic review and meta-analysis. Br. J. Clin. Pharmacol. 63:387-393.

Anderson, B. C. 1998. Cryptosporidiosis in bovine and human health. J. Dairy Sci. 81:3036-3041.

Collick, A. S., E. A. Fogarty, P. E. Zieglar, M. T. Walter, D. D. Bowman, and T. S. Steenhuis. 2006. Survival of Cryptosporidium parvum oocysts in calf housing facilities in the New York City watersheds. J. Environ. Qual. 35:680-687.

Dean, A. G., J. A. Dean, D. Colombier, K. A. Brendel, D. C. Smith, A. H. Burton, R. C. Dicker, K. Sullivan, R. F. Fagan, and T. G. Arner. 1994. Epi Info, Version 6: A Word Processing, Database, and Statistics Program for Epidemiology on Microcomputers. Centers for Disease Control and Prevention, Atlanta, GA.

Elitok, B., O. M. Elitok, and H. Pulat. 2005. Efficacy of azithromycin dihydrate in treatment of cryptosporidiosis in naturally effected dairy calves. J. Vet. Intern. Med. 19:590-593.

Garber, L. P., M. D. Salman, H. S. Hurd, T. Keefe, and J. L. Schlater 1994. Potential risk factors for Cryptosporidium infection in dairy calves. J. Am. Vet. Med. Assoc. 205:86-91.

Harp, J. A., and J. P. Goff. 1995. Protection of calves with a vaccine against Cryptosporidium parvum. J. Parasitol. 81:54-57.

Harp, J. A., and J. P. Goff. 1998. Strategies for the control of Cryptosporidium parvum infection in calves. J. Dairy Sci. 81:289294
Jarvie, B. D., L. A. Trotz-Williams, D. R. McKnight, K. E. Leslie, M. M. Wallace, C. G. Todd, P. H. Sharpe, and A. S. Peregrine. 2005. Effect of halofuginone lactate on the occurrence of Cryptosporidium parvum and growth of neonatal dairy calves. J. Dairy Sci. 88:1801-1806

Jenkins, M. B., L. J. Anguish, D. D. Bowman, M. J. Walker, and W. C. Ghiorse, 1997. Assessment of a dye permeability assay for determination of inactivation rates of Cryptosporidium parvum oocysts. Appl. Environ. Microbiol. 63:3844-3850.

Johnson, J. L., S. M. Godden, T. Molitor, T. Ames, and D. Hagman. 2007. Effects of feeding heat treated colostrum on passive transfer of immune and nutritional parameters in neonatal dairy calves. J. Dairy Sci. 90:5189-5198.

Kaplan, E. L., and P. Meier. 1958. Nonparametric estimation from incomplete observations. J. Am. Stat. Assoc. 53:457-481.

Lefay, D., M. Naciri, P. Poirier, and R. Chermette. 2001. Efficacy of halofuginone lactate in the prevention of cryptosporidiosis in suckling calves. Vet. Rec. 148:108-112.

Moore, D. A., R. E. Atwill, J. H. Kirk, D. Brahmbhatt, L. Herrera Alonso, L. Hou, M. D. Singer, and T. D. Miller. 2003. Prophylactic use of decoquinate for infections with Cryptosporidium parvum in experimentally challenged neonatal calves. J. Am. Vet. Med. Assoc. 223:839-845.

Moore, D. A., and D. H. Zeman. 1991. Cryptosporidiosis in neonatal calves: 277 cases (1986-1987). J. Am. Vet. Med. Assoc. 198:19691971

Nydam, D. V., G. Lindergard, F. Santucci, S. L. Schaaf, S. E. Wade, and H. O. Mohammed. 2005. Risk of infection with Cryptosporidium parvum and Cryptosporidium hominis in dairy cattle in the New York City watershed. Am. J. Vet. Res. 66:413-417.

Nydam, D. V., and H. O. Mohammed. 2005. Quantitative risk assessment of Cryptosporidium species infection in dairy calves. J. Dairy Sci. 88:3932-3943.

Nydam, D. V., and S. A. Peregrine. 2005. Present and future control of cryptosporidiosis in cattle. Pages 15-18 in Proc. 38th Annu. Conv. Am. Assoc. Bovine Pract., Salt Lake City, UT.

O'Handley, R. M., C. Cockwill, T. A. McAllister, M. Jelinski, D. W. Morck, and M. E. Olson. 1999. Duration of naturally acquired giardiosis and cryptosporidiosis in dairy calves and their association with diarrhea. J. Am. Vet. Med. Assoc. 214:391-396.

Peeters, J. E., I. Villacorta, M. Naciri, and E. Vanopdenbosch. 1993. Specific serum and local antibody responses against Cryptosporidium parvum during medication of calves with halofuginone lactate. Infect. Immun. 61:4440-4445.

Rosner, B. A. 1986.Nonparametric methods. Pages 278-293 in Fundamentals of Biostatistics. Duxbury Press, Pacific Grove, CA.

Rossignol, J. F. 2006. Nitazoxanide in the treatment of acquired immune deficiency syndrome-related cryptosporidiosis: Results of the United States compassionate use program in 365 patients. Aliment. Pharmacol. Ther. 24:887-894.

Saini, P. K., G. Ransom, and A. M. McNamara. 2000. Emerging public health concerns regarding cryptosporidiosis. J. Am. Vet. Med. Assoc. 217:658-663.

Trotz-Williams, L. A., S. W. Martin, K. E. Leslie, T. Duffield, D. V. Nydam, and A. S. Peregrine. 2007. Calf-level risk factors for neonatal diarrhea and shedding of Cryptosporidium parvum in Ontario dairy calves. Prev. Vet. Med. 82:12-28.

Trotz-Williams, L. A., S. W. Martin, K. E. Leslie, T. Duffield, D. V. Nydam, and A. S. Peregrine. 2008. Association between management practices and within-herd prevalence of Cryptosporidium parvum shedding on dairy farms in southern Ontario. Prev. Vet. Med. 83:11-23.

Viel, H., H. Rocques, J. Martin, and C. Chartier. 2007. Efficacy of nitazoxanide against experimental cryptosporidiosis in goat neonates. Parasitol. Res. 102:163-166.

Viu, M., J. Quílez, C. Sánchez-Acedo, E. del Cacho, and F. LópezBernad. 2000. Field trial on the therapeutic efficacy of paromomycin on natural Cryptosporidium parvum infections in lambs. Vet. Parasitol. 90:163-170. 